\title{
Frege on Sense and Reference
}

Ted Sider

Phil Language

Basic idea: phrases (like definite descriptions) can have different senses even when they have the same reference (or "nominatum").

\section{Sense}

Now it is plausible to connect with a sign (name, word combination, expression) not only the designated object, which may be called the nominatum of the sign, but also the sense (connotation, meaning) of the sign in which is contained the manner and context of presentation... The nominata of 'evening star' and 'morning star' are the same but not their senses...

The sense of a proper name is grasped by everybody who is sufficiently familiar with the language or totality of designations of which the proper name is a part...

The regular connection between a sign, its sense and its nominatum is such that there corresponds a definite sense to the sign and to this sense there corresponds again a definite nominatum; whereas not one sign only belongs to one nominatum (object). In different languages, and even in one language, the same sense is represented by different expressions. It is true, there are exceptions to this rule. Certainly there should be a definite sense to each expression in a complete configuration of signs, but the natural languages in many ways fall short of this requirement. We must be satisfied if the same word, at least in the same context, has the same sense. It can perhaps be granted that an expression has a sense if it is formed in a grammatically correct manner and stands for a proper name. But as to whether there is a denotation corresponding to the connotation is hereby not decided. The words 'the heavenly body which has the greatest distance from the earth' have a sense; but it is very doubtful as to whether they have a nominatum...

Both the nominatum and the sense of a sign must be distinguished from the associated image... The image is subjective; the image of one person is not that of another... [A sense] may well be common property of many and is therefore not a part or mode of the single person's mind; for it cannot well be denied that mankind possesses a common treasure of thoughts which is transmitted from generation to generation. (pp. 2 I 8-19) 
Key ideas:

Senses are modes of presentation Senses are rules that determine a reference given how the world is. If, for example, you say 'the morning star', the sense is this rule for determining the referent: find the object that is a star (well, heavenly body) and rises in the morning. The sense of a definite description will thus involve the sense of the predicate contained in that definite description.

Sense is linguistic meaning Competent speakers grasp the senses of their words

Sense and ambiguity If a word is meaningful and unambiguous then it has a single sense

Sense determines reference If two expressions have the same sense then they have the same referent (if they have referents at all)

Reference does not determine sense Two expressions with the same referent need not have the same sense ('the morning star' and 'the evening star')

Sense without reference An expression can have a sense without having any referent at all ('the heavenly body which has the greatest distance from the earth')

Senses are public Senses aren't ideas or images which are essentially private.

Terminology: a sign expresses its sense, and designates its referent. This referent is the object that is presented by the sense.

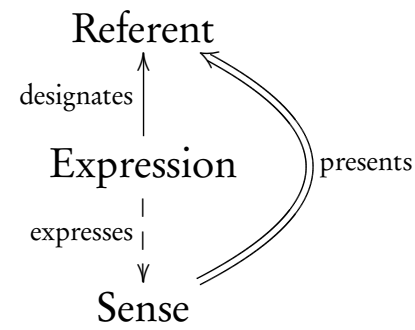




\section{Sense and reference for sentences}

The sense of a sentence is a "thought" (not a mental object though). Think of thoughts as made up of the senses of the words in the sentence.

The referent of a sentence is its truth value—either "The True" or "The False".

\section{Frege's Principles of Compositionality}

i) if the expressions in two sentences have the same senses (and are put together in the same way) then those sentences have the same sense

ii) if the expressions in two sentences have the same referents (and are put together in the same way) then those sentences have the same referent

"the sense/referent of the whole is a function of the senses/referents of the parts"

\section{Sense and reference for predicates}

The referent of a predicate is its extension. The sense of a predicate is an "attribute".

\section{Sense and reference for subjects}

The referent of a definite descriptions is just what you'd think; and the sense is "directions" for picking the referent out, the "manner of presentation" of the referent (contains the sense of the predicate in the definite description).

The referent of a proper name like 'Aristotle' is again the obvious-the man, Aristotle. As for the sense, it's basically the sense of a definite description:

In the case of genuinely proper names like 'Aristotle' opinions as regards their sense may diverge. As such may, e.g., be suggested: Plato's disciple and the teacher of Alexander the Great. Whoever accepts this sense will interpret the meaning of the statement "Aristotle was born in Stagira" differently from one who interpreted the sense of 'Aristotle' as the Stagirite teacher of Alexander the Great. As long as the nominatum remains the same, these fluctuations in sense are tolerable. But they should be avoided in the system of a demonstrative science and should not appear in a perfect language. (p. 228) 


\section{Frege diagrams}

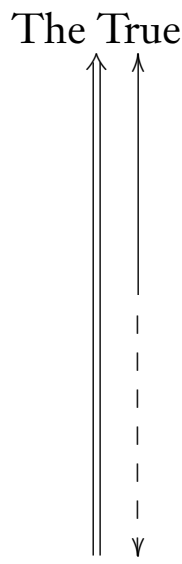

$\mathrm{TPH}$

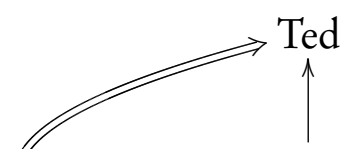

The teacher of phil language

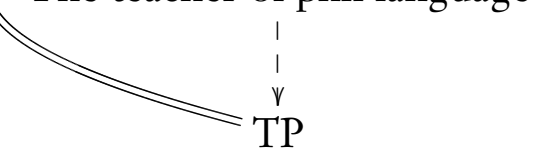

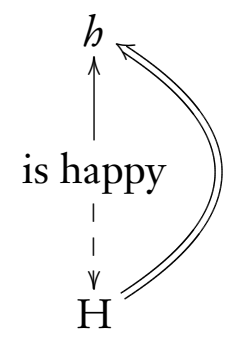

TP: the sense of 'the teacher of phil language'

$\mathrm{H}$ : $\quad$ the attribute of happiness

TPH: the thought that the teacher of phil language is happy

$h: \quad$ the set of happy people

\section{Solution to apriority and analyticity problem}

(I) and (2) have different senses-express different thoughts. Apriority and analyticity are functions of the sense, not the referent, of a sentence.

\section{Indirect discourse}

Apparent problem: (3) and (4) are the same except for a substitution of 'Scott' for 'The author of Waverly'. So, given compositionality of reference, they should have the same referent-and so, the same truth value.

Solution: the Frege shift. Inside propositional attitude verbs, such as 'believed that', 'hoped that', 'said that', etc., sentences and other expressions denote their customary senses. 